\title{
Virtual Prototyping Application using Computer Aided Engineering in Plastic Product Manufacturing
}

\author{
Case Study: \\ Plastic Handle Product using Injection Molding Technology Process in PT. Dharma Polimetal
}

Ahmad Juang Pratama

Dept. of Industrial Engineering, Faculty of Science and Technology,

University of Al Azhar Indonesia, Jl.Sisingamangaraja, Jakarta 12110

email: juang@uai.ac.id

\begin{abstract}
Efficiency in using plastic injection technology, especially within small to medium enterprises, is a very difficult target to achieve. One of the contributing factor to the high cost of using this technology is the trial and error nature of constructing the plastic mold which directly affect machninig cost and longet production cycle time. The trial and error scheme can be significantly reduced and even eliminated with the application of concurrent engineering method supported by virtual prototype based CAD (computer aided design) and CAE (computer aided engineering). Virtual prototype technology gives way to product analysis, design and material optimization, and proses parameter optimization prior to implementing the manufacturing process. Therefore, we can anticipate all potential manufacturing flaws during the design process. It is then expeceted to reduce machning and production process cost, increase product quality to boost up Company's competitive advantage.
\end{abstract}

Keywords - Injection Molding, Virtual Prototype, CAD, CAE, Physical Prototype, Concurrent Engineering, Simulation.

\section{INTRODUCTION}

$\mathrm{M}$ anufacturing process for plastic-based products using injection molding technology has currently been widely spread out nationally among various manufacturing industries such as automotive, packaging, hospital equipment, electornics and many more. This is because the plastic has a light nature, strong and easily formed, anti-rust and resistant to chemicals, have high electrical insulation nature, can be colored and transparent process and cost less. Along with the strict competition among the manufacturers of plastic goods and the desire to meet and satisfy customer needs, the need for product variations increase but with a quick response. The current philosophy is to constantly replace old products with either an improved product or a new variation of the product. This will make the product development cycle shorter and difficult to take advantage in the framework economy of scale. Thus the challenge here is to minimize the internal complexity and its resulting costs without reducing the range of products [1].

During product development, one that has a big contribution in production costs is the need for creating a prototype to be used for the iterative evaluation to provide feedbacks for design modification such as selection of design alternatives, engineering analysis, manufacturing planning and Visualization of a product

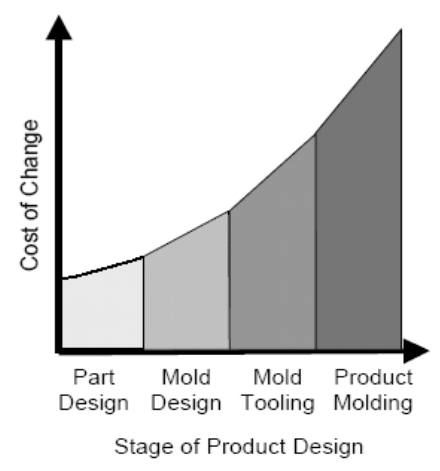

Figure 1. cost change in mold design[2] 


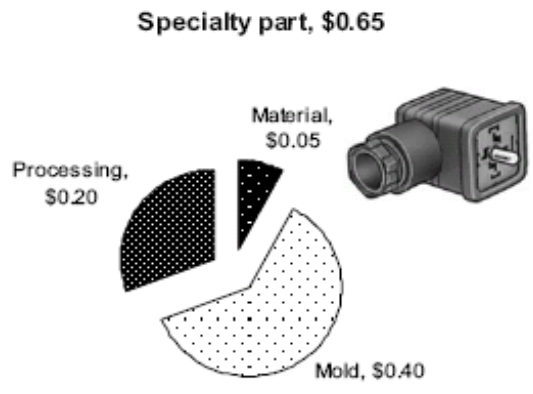

Figure 2. cost factor in mold production [3]

As shown in the picture above, the changes that occur in the new mold making process will affect the cost of which is very high. Meanwhile to be successful to make a mold, there are many factors that must be considered including design, process, and material. In Reality, reworks in the making of a new mold prototype can not be avoided.

Concurrent engineering method using Virtual Prototyping supported by CAD (computer Aided Engineering) and CAE (Computer Aided Engineering) is one of approach to these problems. Virtual Prototyping Tools (CAD and CAE) offer the tremendous advantage of enabling design teams to consider virtually any molding option without incurring the expense associated with physical prototyping. The ability to try new designs or concepts using CAD tool gives the opportunity to eliminate problems before production begin. Additionally, designers can quickly and easily determine the sensitivity of specific molding parameters on the quality and production of the final part. By transferring data from CAD tools to CAE tools designer able to determine the effects of different injection point, gating altervatives, geometric features, and different molding and processing conditions of mold and quality of the final part. Furthermore, the Combination CAD and CAE tools allow these virtual designs to be completed in a matter of days or even hours rather than the weeks associated with physical trial and error.

In this study, one of real plastics mold design design that is consistent to gives defect contributions is redrawing in 3D CAD solid model and then examined using CAE software that employ finite element method. Thus the potential design flaws that will contribute to quality problem can be identified, consequently, unnecessaries modifications can be prevented.
This paper consist of 4 section, Section I gives the Introduction and the background, section II gives the theoretical background about concurrent engineering, virtual prototyping and its relevance. Section III deals with mold design simulation and analysis. Finally, Section IV gives conclusion and the remarks for thi study.

\section{BASIC THEORY}

Concurrent engineering is a business strategy which replaces the conventional product development process with strategy in which tasks are done in parallel and there is an early consideration for every aspect of a product development process. Moreover, product design, testing, manufacturing process planning and logistics are done simultaneously and interactively. Thus, potential problems in the manufacturing, assembly, support, and quality are identified and solved early in the design process.

CAD is solid 3D design tool, while CAE analyze 3D CAD model. Combination these two technology support and increase the speed and quality of Concurent engineering process, because it wouldn't be necessaries to apply all of physical processes in plactics injection product development phase

\subsection{Virtual Prototyping Definition}

Along with the rapid development of design technology, concurrent engineering method is supported by design tools called a virtual prototype. According Wang [6] "Virtual prototype, or digital mock-up, is a computer simulation of a physical product that can be presented, analyzed, and tested from concerned product life-cycle aspects such as design/engineering, manufacturing, service, and recycling as if on a real physical model. The construction and testing of a virtual prototype is called virtual prototyping (VP)."

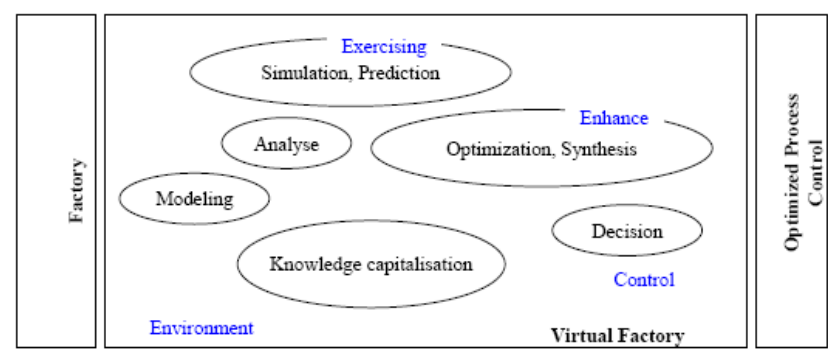

Figure 3. Virtual prototype area [4] 
Virtual prototyping provide quick design iteration process, where problems can be rectified immediately whenever indicated in analisys. More over, solving the problem in VP domain helps to reduce physical prototype costs and time[5]. In engineering product application, Virtual Prototyping is supported by CAD software (Computer Aided Design) for representing geometric information, and CAE software (Computer Aided Engineering) for analyzing design manufacturability, process, material, and behaviour of design. Geometric stereolithography (stl) data from CAD solid modelling software is exported to CAE software to be simulated.

\subsection{Molding Simulation Process}

The product geometry data with stereolithography extension (stl) used by molding simulation software (CAE) had to be generated and imported from independent 3D solid CAD program.

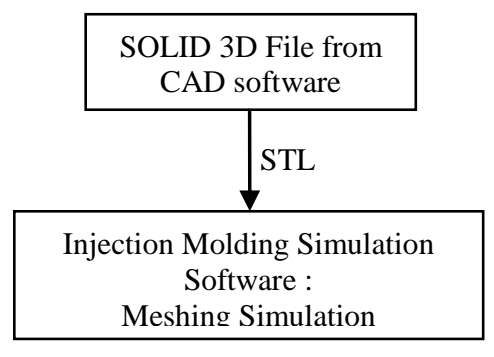

Figure 4. Simulation Process using CAE software

Injection molding simulation programs use the part geometry to define the mold cavity. Once in the simulation program, the STL mesh information is used to generate a compatible plastic melt flow model simulating the mold cavity, i.e., a. finite element mesh. The mesh generation is done automatically or manually by user intervention. In preparation for the simulation, the designer selects an injection point over the desired location. Then, the designer selects from a material database the type of resin to be used. Processing conditions can also be modified by designer. But the database give default values for resin melt temperature and mold temperature are default values recommended for the resin selected. The injection rate may also be automatically computed by the simulation program based on the part geometry and the chosen gating locations (points where the plastic enters the mold cavity). The number and location of gates used to fill a mold cavity have the greatest influence on whether that part can be molded.
Analyzing part while in Virtual prototyping domain give user flexibility to examine a number of gating alternatives to optimize the filling pattern. During the preliminary stages of design, a designer selects a point on the solid model to define a gate. The rapid feedback in these programs makes them easy to use for multiple product design and gating iterations. After a product design is shown to be moldable with a given gating option, a runner system may be added to the mold cavity model to better model the flow of plastic into a cavity that has more than one gate (or one cavity). The addition of runner system would be done with CAD program that generated the product geometry or feature provided by CAE program. Once the plastic filling pattern is balanced, then designers can perform cooling and quality analysis, this is necessary to make the cooling process in mold cavity uniform so the designer can prevent many part quality problem.

\subsection{Simulation Result}

Simulation outputs from CAE is in quantitive value, and is visualized in blue, yellow, and red color on the solid model. The colors represent certaint value that show material flow into the part cavity, cavity pressure, melt-flow temperature and time to freeze. For example; red represent undesired or low values, yellow for transition values, and green for acceptable valuea or condition. After simulation, a report is automatically generated that summarizes key factors of the product design that may have adversely affected molding. Such factors may be large variations in product wall thickness or relatively thin wall thickness for the gating option and material selected, or melt temperature distribution. For a given gate location, the simulation informs the user the filling pattern of the part by displaying the orientation of molecul. If a short shot occurs because of bad gating design, inadequate processing parameter, or poor product design, the software shows the designer the possible causes of the defect and suggestion to fix them. 


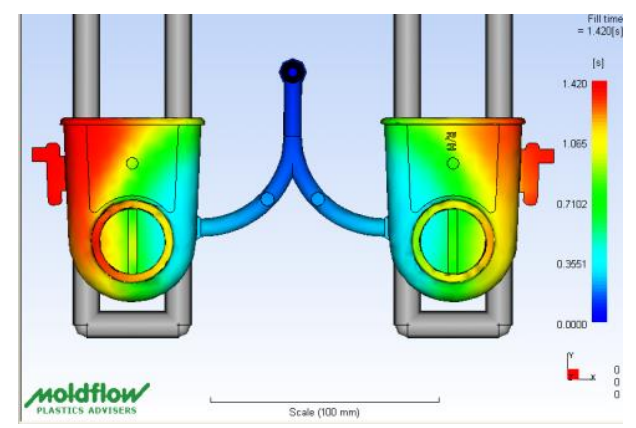

Figure 5. Filling quality visualization

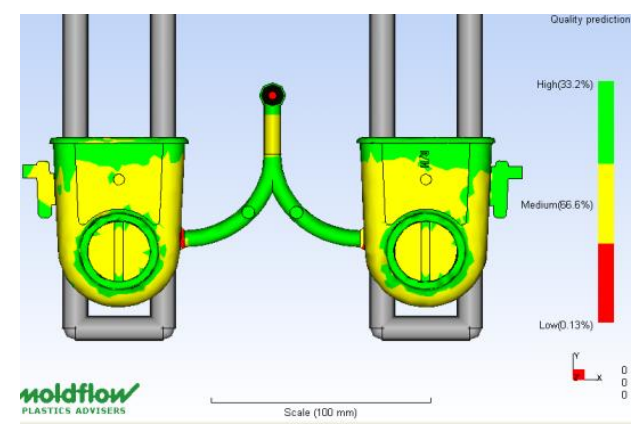

Figure 6. Quality prediction visualization

\section{RESEARCH METHODS}

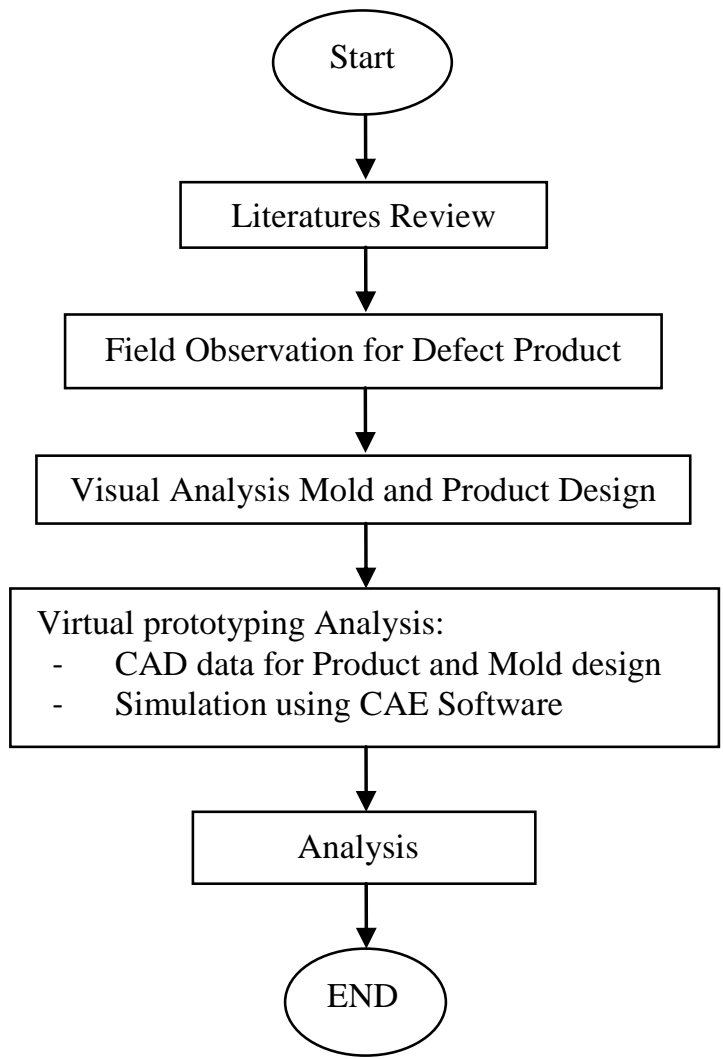

Figure 7. Research Methodology Diagram
This research begin with literatures review and then field analysis for defect product. We choose a product design that has relatively high contribution to number of fail product and it is difficult to be analyzed by operator. After unsolved product design problem was found, we conducted visual analysis of Product and Mold design. The next step, we gather the 3D CAD data and then simulate it using CAE software. In the final step we compare physical product defect with virtual prototyping result to see whether the simulation gives near prediction of real product defects.

\section{RESULT AND DISCUSSION}

An example of a student group project illustrates what can be achieved with CAE simulation software. In this case a student group investigated real plastic part mold design. Figure 8 show the plastics product, figure 9 and figure 10 show the real physical mold parts

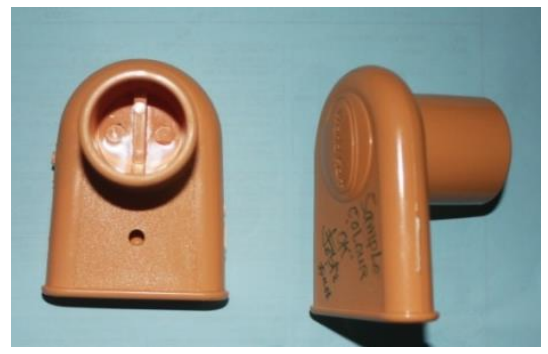

Figure 8. Plastic Handle

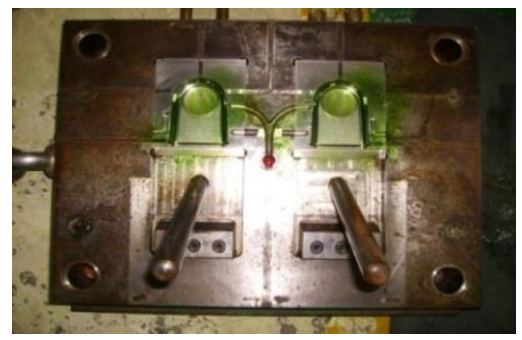

Figure 9. Mold cavity

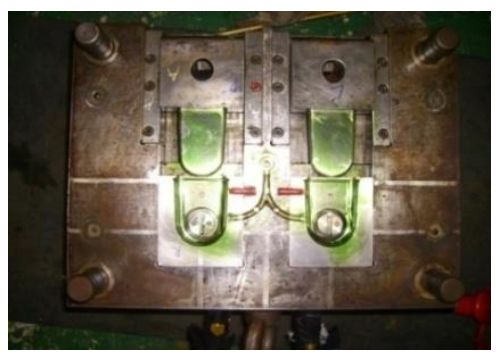

Figure 10. Mold cor 


\subsection{Simulation Result Versus Reality}

Simulation result above show quality level of original Plastics Handle is not very good. Percentage for high quality level has only $33.2 \%$ while the percentage of medium $66.6 \%$ and low $0.13 \%$. The level of high quality is not high indicates that Plastics Handle will often have quality problems.

As seen from Figure 12, temperature variance result show yellow area have higher temperature than other yellow green area. This slower cooling rate is caused by distance between part and bottom cooling channel is relatively longger. This can be also interpreted as the need of additional top cooling channel. This is also cause by different part thickness especially on the top of thicker tube hole.

More over, simulation also shows different cooling time variance, on the red area (thicker tube hole), it takes about $18.66 \mathrm{~s}$ to freeze. Supported from temperature variance result from figure 13 , the designer was able to find fact that the original Plastics Handle cooling rate is not uniform. One important key to achieve good quality injection molding part is uniformity of cooling. In reality, the defect area was found on yellow area of the part exactly as predicted by simulation software

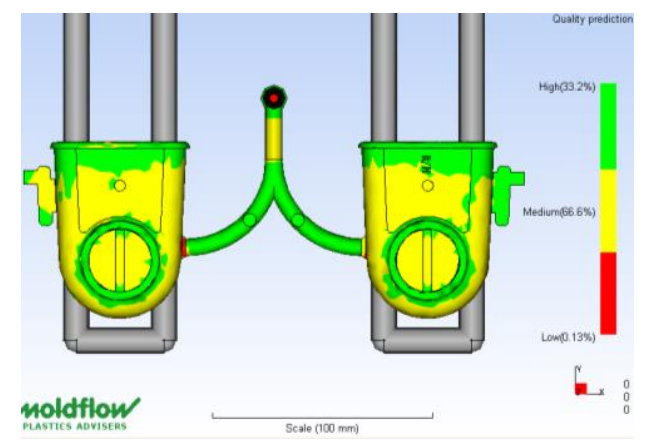

Figure 11. quality prediction

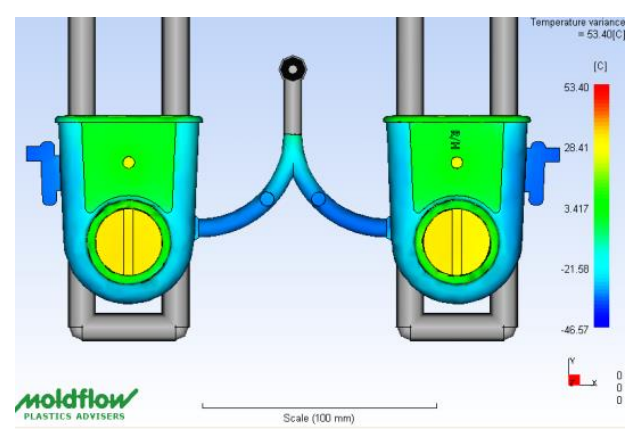

Figure 12. Temperature Variance

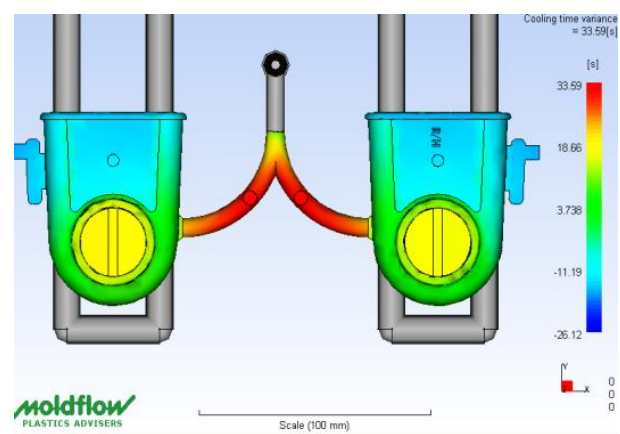

Figure 13. Cooling time variance

\subsection{Suggestion for Mold Design Improvement and Design Verification.}

Based on the finding facts in the previous simulation, user was able to determine cause of defect parts, and propose solution to fix this quality problem. By considering material cost, tooling cost, time, and technical difficulty level, a simple additional cooling channel and buffel modification was proposed.

Before implementing the design modification in real physical mold, one needs to verify the design in virtual prototyping area $o$ ensure that the proposed modification is appropriate. Thus prevent modification in physical mold. Consequently, increase the product quality, and prevent modification in physical mold.

After cooling system modification, temperature variance result from figure 15 show lower temperature variance. As seen, yellow area in figure 12 turn to be light blue area in figure 15 . In this case, additional cooling channel give more effective cooling rate, and give better cooling uniformity degree

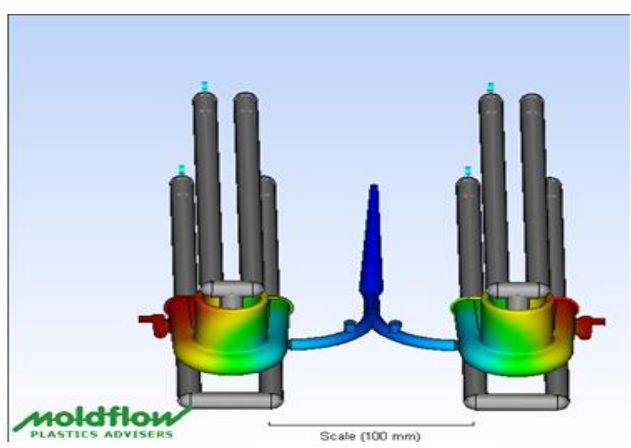

Figure 14. proposed solution 
Figure 16 also shows additional cooling channel affects lower cooling time variance which lead to better cooling characteristics and better part quality prediction

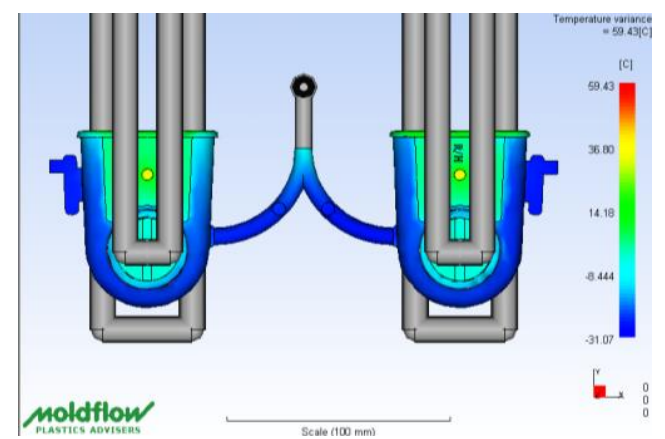

Figure 15. Temperature variance

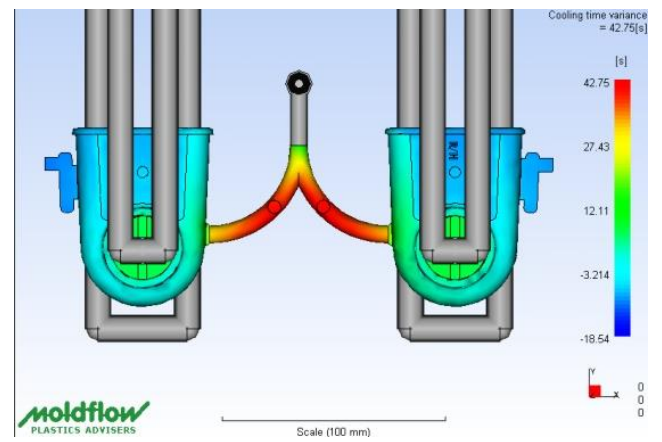

Figure 16. Cooling time variance

\subsection{Rapid iterative design and simulation speed}

In this study we had tried many design alternatives to find better combination of design, process parameters, plastics and mold material. Based on our expecience, Sofware calculation can be done within minutes or hours but it depends on dimension of mold design to be simulated, accuracy setting, and hardware capability. We use simulation sofware that have default setting of accuracy, assume at medium level of accuracy and use only medium computer speed. However, this condition still give us advantage to enjoy good calculation speed, and be able to do several simulation tasks a day compare to do trial and error in domain of physical prototyping that probably take weeks or months.

\section{CONCLUSIONS}

Defects that happen on real product are the same as one that indicated in simulation result. By knowing root design problem in earlier design process, actually such design flaw can be prevented from the beginning of production process. Before the digital prototyping implementation, mold design improvements are implemented on the expert experience basis. This is very difficult because no exact quantitive value.

Virtual Prototyping using CAD and CAE sofware is powerful tool for predicting and analyzing manufacturability, quality, of plastics molding injection part prior to production. Potential problems in the manufacturing, assembly, support, and quality are identified and solved early in the design process. Virtual prototyping application in the context of plastics product using injection molding technologies process give greater flexibility to cross the border of mold design innovation. Supported by fairly good computer speed, the simulation software is able to give faster analysis compared to trial and error scheme of real physical prototyping to find the best and cheapest design alternatives. Moreover, further study should investigate the impact of Virtual prototyping application in term of cost and time saving, also its impact knowledge management in Concurrent Engineering Method.

\section{REFERENCES}

[1] T. Kipp and D. Krause, "Design for variety Efficient support for design engineers" INTERNATIONAL DESIGN CONFERENCE DESIGN 2008, Dubrovnik - Croatia, pp. 1-8, May 2008.

[2] L. Reifschneider, "Teaching Design for Manufacturability with Desktop Computer-Aided Analysis", Journal of Industrial Technology, Vol 16, No. 3, pp.2, May 2000

[3] David O. Kazmer, Injection Mold Design Engineering, Karl Hanser Verlag, Munich, 2007

[4] Philippe Dépincé, Damien Chablat, Peer-Oliver Woelk. "Virtual manufacturing Tools for improving design and production, Institute of Production Engineering and Machine-Tools“, pp.3, Hannover 2002.

[5] Silva Bartolo et all, "The feasibility of customer evaluation of products using virtual prototyping technologies", Proceedings of the 3rd International Conference on Advanced Research in Virtual and Rapid Prototyping, Leira Portugal, pp.704, September 2007.

[6] Wang G.G. "Definiton and review of virtual prototyping", Journal of Computing and Information Science In engineering. Sept 2002, Vol 2, No. 3, pp. 232-236, September 2002 